\title{
One fewer solution to the cosmological lithium problem
}

\author{
O. S. Kirsebom ${ }^{1,2, *}$ and B. Davids ${ }^{1}$ \\ ${ }^{1}$ TRIUMF, Vancouver, BC V6T 2A3, Canada \\ ${ }^{2}$ Department of Physics and Astronomy, Aarhus University, 8000 Aarhus C, Denmark
}

\begin{abstract}
Data from a recent ${ }^{9} \mathrm{Be}\left({ }^{3} \mathrm{He}, t\right){ }^{9} \mathrm{~B}$ measurement are used to rule out a possible solution to the cosmological lithium problem based on conventional nuclear physics.
\end{abstract}

PACS numbers: 26.35.+c, 98.80.Ft

The primordial abundance of ${ }^{7} \mathrm{Li}$ inferred from observational data is roughly a factor of three below the abundance predicted by the standard theory of big bang nucleosynthesis (BBN) 1] using the baryon-to-photon ratio $\eta=6.19(15) \times 10^{-10}[2]$ determined mainly from measurements of the cosmic microwave background radiation. In contrast, there is good agreement for ${ }^{2} \mathrm{H}$ and ${ }^{4} \mathrm{He}$. Taking into account the estimated uncertainties on the observationally inferred and the theoretically deduced ${ }^{7} \mathrm{Li}$ abundances, the significance of the discrepancy is $4.2 \sigma-5.3 \sigma[3]$. This constitutes one of the important unresolved problems of present-day astrophysics and is termed the cosmological lithium problem. Among other possibilities, the discrepancy could be due to new physics beyond the Standard Model of particle physics [4], errors in the observationally inferred primordial lithium abundance $^{1}$, or incomplete nuclear physics input for the BBN calculations. The present paper addresses the last possibility.

In standard BBN theory, assuming $\eta=6.19(15) \times$ $10^{-10}$, most ${ }^{7} \mathrm{Li}$ is produced in the form of ${ }^{7} \mathrm{Be}$. Only much later, when the Universe has cooled sufficiently for nuclei and electrons to combine into atoms, does ${ }^{7} \mathrm{Be}$ decay to ${ }^{7} \mathrm{Li}$ through electron capture. The temperature range of ${ }^{7} \mathrm{Be}$ production is $T \simeq 0.3-$ $0.6 \mathrm{GK}$, where the main mechanism for ${ }^{7} \mathrm{Be}$ production is ${ }^{3} \mathrm{He}(\alpha, \gamma){ }^{7} \mathrm{Be}$ while the main mechanism for ${ }^{7} \mathrm{Be}$ destruction is ${ }^{7} \mathrm{Be}(n, p){ }^{7} \mathrm{Li}$ followed by ${ }^{7} \mathrm{Li}(p, \alpha){ }^{4} \mathrm{He}$. The rates of these reactions as well as the reactions that control the supply of neutrons, protons, ${ }^{3} \mathrm{He}$, and $\alpha$ particles are known with better than $10 \%$ precision at BBN temperatures [6], resulting in an uncertainty of only $13 \%$ on the calculated ${ }^{7} \mathrm{Li}$ abundance 3 ].

A recent theoretical paper [7] explores the possibility of enhancing ${ }^{7} \mathrm{Be}$ destruction through resonant reactions with $p, d, t,{ }^{3} \mathrm{He}, \alpha$, leading to compound states in ${ }^{8} \mathrm{~B},{ }^{9} \mathrm{~B}$, ${ }^{10} \mathrm{~B},{ }^{10} \mathrm{C},{ }^{11} \mathrm{C}$, respectively. The paper concludes that, of the known excited states in these isotopes [9, 10], only the $16.8 \mathrm{MeV}$ state in ${ }^{9} \mathrm{~B}$ has the potential to significantly influence ${ }^{7} \mathrm{Be}$ destruction ${ }^{2}$. (Note that in Ref. [7] this

\footnotetext{
*Corresponding author: oliverk@triumf.ca

${ }^{1}$ Lithium may be destroyed in metal-poor stars through diffusion and turbulent mixing [5].

${ }^{2}$ Ref. [8] offers a more optimistic view, but only by adopting a somewhat flexible approach to basic principles of nuclear physics.
}

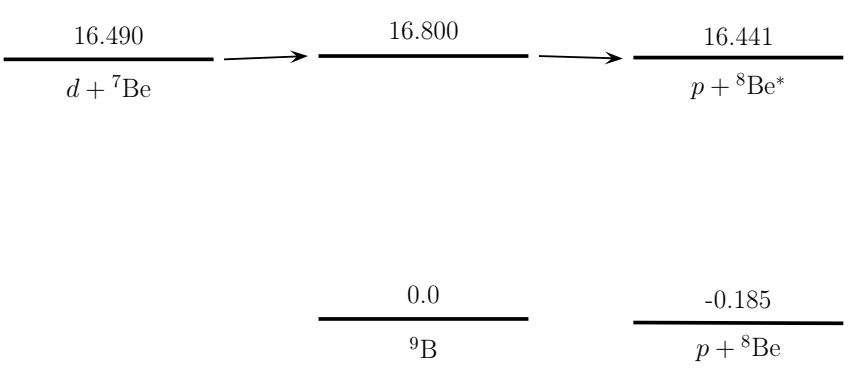

FIG. 1: Schematic illustration of the proposed ${ }^{7}$ Be destruction mechanism, $d+{ }^{7} \mathrm{Be} \rightarrow{ }^{9} \mathrm{~B}^{*} \rightarrow p+{ }^{8} \mathrm{Be}^{*}$. The energies are in $\mathrm{MeV}$ relative to the ground state of ${ }^{9} \mathrm{~B}$. Subsequently, ${ }^{8} \mathrm{Be}^{*}$ breaks up into two $\alpha$ particles.

state is referred to as the $16.7 \mathrm{MeV}$ state.) The proposed destruction mechanism is shown schematically in Fig. 1. The $16.8 \mathrm{MeV}$ state in ${ }^{9} \mathrm{~B}$ is formed by the fusion of ${ }^{7} \mathrm{Be}$ with a deuteron and decays by proton emission to a highly excited state in ${ }^{8} \mathrm{Be}, 16.626 \mathrm{MeV}$ above the ground state, which subsequently breaks up into two $\alpha$ particles. (The last step is not shown in the figure.) The reason why the decay must proceed by proton emission to the $16.626 \mathrm{MeV}$ state in ${ }^{8} \mathrm{Be}$ and not e.g. the ground state will be explained below.

The reaction rate depends critically on the resonance energy, $E_{r}$, i.e. the energy of the $16.8 \mathrm{MeV}$ state relative to the $d+{ }^{7} \mathrm{Be}$ threshold at $S_{d}=16.4901(10) \mathrm{MeV}[9]$ : if too far above the threshold, the tunneling process will be too slow at BBN temperatures. Furthermore, for the proposed destruction mechanism to be efficient, the 16.8 MeV state must have an appreciable width, $\Gamma_{d}$, for being formed in the $d+{ }^{7} \mathrm{Be}$ channel, but also an appreciable width, $\Gamma-\Gamma_{d}$, for not decaying back to $d+{ }^{7} \mathrm{Be}$. The energetically allowed decay modes competing with deuteron emission are: $\gamma, \mathrm{p}, \alpha$, and ${ }^{3} \mathrm{He}$. However, $\gamma$ and ${ }^{3} \mathrm{He}$ can safely be neglected. A deuteron width, $\Gamma_{d}$, of the required magnitude can only be realized if the 16.8 MeV state is not too close to the threshold. The analysis of Ref. [7] shows that the cosmological lithium problem can be resolved provided $E_{r} \simeq 170-220 \mathrm{keV}$, $\Gamma_{d} \simeq 10-40 \mathrm{keV}$, and $\Gamma-\Gamma_{d} \simeq \Gamma_{d}$. At the time Ref. [7] was written, the known properties of the $16.8 \mathrm{MeV}$ state did not contradict these requirements: The $16.8 \mathrm{MeV}$ state had been observed in two experiments [11, 12]. Its energy had been determined to be $16.7 \mathrm{MeV}$ with an un- 
certainty of $100 \mathrm{keV}$, and only an upper limit of $100 \mathrm{keV}$ existed on its total width. Its spin and parity had not been determined, though a tentative $5 / 2^{+}$assignment had been made [13] based on comparison to the mirror nucleus, ${ }^{9} \mathrm{Be}$. No information existed on its decay properties.

As noted in Ref. 7], the simultaneous requirement of $E_{r} \simeq 170-220 \mathrm{keV}$ and $\Gamma_{d} \simeq 10-40 \mathrm{keV}$ is physically possible, but implies some rather special properties for the $16.8 \mathrm{MeV}$ state: a reduced deuteron width comparable to the Wigner limit and a very large channel radius of at least $9 \mathrm{fm}$. In addition, the proposed destruction mechanism could only be reconciled with the direct measurement of Ref. [14] with considerable difficulty: the proton and $\alpha$ decay of the $16.8 \mathrm{MeV}$ state had to be dominated by a single proton-decay branch to the $16.626 \mathrm{MeV}, 2^{+}$state in ${ }^{8} \mathrm{Be}$, because decays to the lower-lying states in ${ }^{8} \mathrm{Be}$ would have produced protons of sufficient energy to be detected by the experimental setup of Ref. [14].

Slightly above the $16.626 \mathrm{MeV}$ state, at $16.922 \mathrm{MeV}$, lies another $2^{+}$state in ${ }^{8} \mathrm{Be}$. The two are nearly maximally mixed in isospin $(I)$ [15] and are often referred to as the $2^{+}$doublet. The structure of the $16.626 \mathrm{MeV}$ state is primarily that of a $1 p_{1 / 2}$ proton orbiting a ${ }^{7} \mathrm{Li}$ core in its ground state, and the structure of the $16.922 \mathrm{MeV}$ state is primarily that of a $1 p_{1 / 2}$ neutron orbiting a ${ }^{7} \mathrm{Be}$ core in its ground state [16]. The analysis in Ref. [13] suggests that the structure of the $16.8 \mathrm{MeV}$ state in ${ }^{9} \mathrm{~B}$ is primarily that of a $2 s_{1 / 2}$ proton orbiting the $I=1$ component of the $2^{+}$doublet. As noted in Ref. 7], this provided reason to think that the overlap between the 16.8 MeV state in ${ }^{9} \mathrm{~B}$ and $p+{ }^{8} \mathrm{Be}^{*}$ might indeed be considerably larger for the $16.626 \mathrm{MeV}$ state than for any of the lower-lying states in ${ }^{8} \mathrm{Be}$.

The nuclear physics input for BBN calculations has recently been reexamined in Ref. [17], which includes new reactions, studies the potential effects of reactions for which data do not exist, studies the effects of non-thermal particles (highly energetic particles produced in exothermic reactions), in particular neutrons which take much longer to thermalize than charged particles, and includes thermal excitation of the first excited states in ${ }^{7} \mathrm{Li}$ and ${ }^{7}$ Be. Ref. [17] concludes that there is little chance of solving the cosmological lithium problem with conventional nuclear physics, but retains the destruction mechanism proposed by Ref. [7] as an "alluring" possibility.

A very recent paper [18] reports on a new ${ }^{9} \mathrm{Be}\left({ }^{3} \mathrm{He}, t\right){ }^{9} \mathrm{~B}$ measurement performed with the purpose of studying Gamow-Teller transition strengths in the $A=9$ system. The beam energy was $140 \mathrm{MeV} /$ nucleon, and tritons were detected in a high-resolution spectrometer at scattering angles around $0^{\circ}$. The excitation energy resolution achieved was $30 \mathrm{keV}$. The $16.8 \mathrm{MeV}$ state is strongly excited, and its energy and width are determined to be $16.800(10) \mathrm{MeV}$ and $81(5) \mathrm{keV}$, respectively, in good agreement with the two previous experiments. The nearby $J^{\pi}=1 / 2^{-}, I=3 / 2$ state at $17.076(4) \mathrm{MeV}$ is also strongly excited and its energy is well-known from its $\gamma$ decay to the ground state. This gives strong confidence in the new energy determination. It is also worth noting that the observed angular distribution of the $16.8 \mathrm{MeV}$ state is consistent with the proposed $5 / 2^{+}$assignment.

To assess the consequence of the new experimental data for the proposed destruction mechanism, we employ the standard Kawano/Wagoner BBN code [19, 20]. We modify the ${ }^{7} \mathrm{Be}(d, p)$ reaction rate by adding the extra term [21]

$$
\begin{aligned}
N_{A}\langle\sigma v\rangle= & N_{A}\left(8 / \pi \mu_{27}\right)^{1 / 2}(k T)^{-3 / 2} \\
& \times \int_{0}^{\infty} E \sigma(E) \exp (-E / k T) \mathrm{d} E,
\end{aligned}
$$

where $N_{A}$ is Avogadro's constant, $\mu_{27}$ is the reduced mass, $k$ is Boltzmann's constant, $T$ is the temperature, $E$ is the relative kinetic energy, and $\sigma(E)$ is the cross section for $d+{ }^{7} \mathrm{Be} \rightarrow{ }^{9} \mathrm{~B}^{*} \rightarrow p+{ }^{8} \mathrm{Be}^{*}$, given by the singlechannel, single-level approximation of the $R$-matrix theory [22]:

$$
\sigma(E)=\pi \lambda^{2} \omega \frac{\Gamma_{d}\left(\Gamma-\Gamma_{d}\right)}{\left(E-E_{r}-\Delta\right)^{2}+(\Gamma / 2)^{2}},
$$

where $\lambda=\hbar / p=\hbar /\left(2 \mu_{27} E\right)^{1 / 2}$, and $\omega$ is a statistical weight factor that depends on the spins involved,

$$
\omega=\frac{2 J+1}{\left(2 j_{1}+1\right)\left(2 j_{2}+1\right)}=\frac{2 \times \frac{5}{2}+1}{(2 \times 1+1)\left(2 \times \frac{3}{2}+1\right)}=0.5,
$$

where $J=5 / 2$ is the (assumed) spin of the $16.8 \mathrm{MeV}$ state, $j_{1}=1$ is the spin of the deuteron, and $j_{2}=3 / 2$ is the spin of ${ }^{7} \mathrm{Be}$. Furthermore, $\Gamma=\Gamma_{\gamma}+\Gamma_{p}+\Gamma_{d}+$ $\Gamma_{{ }^{3} \mathrm{He}}+\Gamma_{\alpha}$ is the total width. We assume $\Gamma_{\gamma}, \Gamma_{{ }^{3} \mathrm{He}}$, and $\Gamma_{\alpha}$ to be negligible and $\Gamma_{p}$ to be dominated by the decay to the $16.626 \mathrm{MeV}$ state in ${ }^{8} \mathrm{Be}$. As the $16.8 \mathrm{MeV}$ state is located close to threshold, the energy dependence of the deuteron width must be taken into account [23]:

$$
\Gamma_{d}=2 P_{\ell=1}(E) \gamma_{d}^{2} .
$$

Similarly, for the proton width:

$$
\Gamma_{p}=2 P_{\ell=0}\left(E^{\prime}\right) \gamma_{p}^{2}
$$

where $P_{\ell}$ is the penetrability, $\ell$ is the orbital angular momentum, $\gamma_{d}\left(\gamma_{p}\right)$ is the deuteron (proton) reduced width, and $E^{\prime}$ is the $p+{ }^{8} \mathrm{Be}^{*}$ relative kinetic energy,

$$
E^{\prime}=E+S_{d}-S_{p}-16.626 \mathrm{MeV}
$$

with $S_{p}=-0.1851(10) \mathrm{MeV}$ [9]. We note that Eq. (2) is only approximately valid, as it assumes that the width of the $16.626 \mathrm{MeV}$ state in ${ }^{8} \mathrm{Be}$ can be neglected, whereas the state actually has a considerable width of 108.1(5) keV [9] with an asymmetric line shape owing to interference with the $16.922 \mathrm{MeV}$ state. Still, the approximation is adequate for the present analysis. Finally, the shift, $\Delta$, is calculated as

$$
\Delta=-\left(S_{\ell=1}(E)-B\right) \gamma_{d}^{2}-\left(S_{\ell=0}\left(E^{\prime}\right)-B^{\prime}\right) \gamma_{p}^{2},
$$




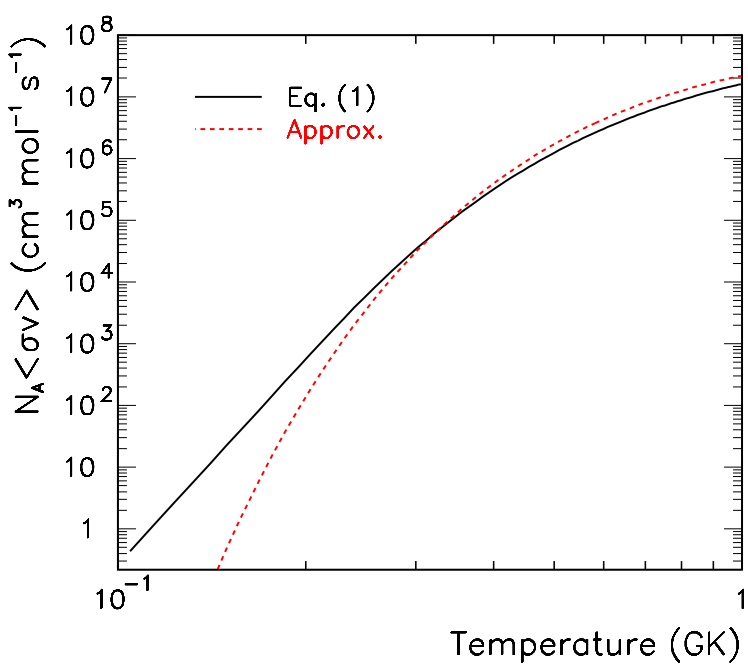

FIG. 2: Maximal resonant contribution of the $16.8 \mathrm{MeV}$ state in ${ }^{9} \mathrm{~B}$ to the ${ }^{7} \mathrm{Be}(d, p)$ reaction rate calculated from Eq. (1) versus temperature. For comparison, the rate calculated using the narrow resonance approximation is also shown.

where $S_{\ell}$ is the shift function, and the boundary conditions are $B=S_{\ell=1}\left(E_{r}\right)$ and $B^{\prime}=S_{\ell=0}\left(E_{r}^{\prime}\right)$. The definitions of $P_{\ell}$ and $S_{\ell}$ are given in Ref. [22]. To evaluate $P_{\ell}$ and $S_{\ell}$, suitable channel radii, $a_{27}$ and $a_{18}$, must be chosen for the formation and destruction channel.

Relying on the data from the new ${ }^{9} \mathrm{Be}\left({ }^{3} \mathrm{He}, t\right){ }^{9} \mathrm{~B}$ measurement [18], we use $E_{r}=310(10) \mathrm{keV}$ for the resonance energy and $\Gamma^{0}=81(5) \mathrm{keV}$ for the total width. The superscript 0 refers to the value at resonance energy, i.e. at $E=E_{r}$. To maximize the reaction rate, we chose $\gamma_{d}$ and $\gamma_{p}$ such that $\Gamma_{d}^{0}=\Gamma_{p}^{0}=0.5 \Gamma^{0}$. We do not have complete liberty in our choice of $\gamma_{d}$ and $\gamma_{p}$ as they should not exceed the corresponding Wigner limits, $\gamma_{\mathrm{W}, d}^{2}=3 \hbar^{2} /\left(2 \mu_{27} a_{27}^{2}\right)$ and $\gamma_{\mathrm{W}, p}^{2}=3 \hbar^{2} /\left(2 \mu_{18} a_{18}^{2}\right)$. We find that a permissible choice of $\gamma_{d}$ only exists for $a_{27}>6.5 \mathrm{fm}$, whereas a permissible choice of $\gamma_{p}$ exists for essentially any value of $a_{18}$. For the present calculation, we chose $a_{27}=7 \mathrm{fm}$ and $a_{18}=5 \mathrm{fm}$, yielding $\gamma_{d}^{2} / \gamma_{\mathrm{W}, d}^{2}=0.93$ and $\gamma_{p}^{2} / \gamma_{\mathrm{W}, p}^{2}=0.09$. As argued in Ref. [7], $a_{27}=7 \mathrm{fm}$ represents a physically plausible choice of channel radius. The temperature dependence of the reaction rate is shown in Fig. 2. For comparison, we also show the rate obtained in the narrow resonance approximation. We find that the reduction in ${ }^{7} \mathrm{Li}$ abundance caused by the inclusion of the resonant contribution of the $16.8 \mathrm{MeV}$ state in ${ }^{9} \mathrm{~B}$ to the ${ }^{7} \mathrm{Be}(d, p)$ reaction rate is at most $3.5(8) \%$. This result is essentially independent of the choice of channel radii. The quoted uncertainty mainly reflects the $10 \mathrm{keV}$ uncertainty on the energy determination of the $16.8 \mathrm{MeV}$ state with a small contribution $(0.2 \%)$ from the $5 \mathrm{keV}$ uncertainty on the width determination.

We stress that the assumption of a dominant protondecay branch to the $16.626 \mathrm{MeV}$ state is, by no means, important to the conclusion of the present analysis. If the decay is assumed to proceed by proton emission to lower-lying states in ${ }^{8} \mathrm{Be}$ or $\alpha$ emission to ${ }^{5} \mathrm{Li}$, a similar reduction in ${ }^{7} \mathrm{Li}$ abundance is obtained. The assumption of a dominant proton-decay branch to the $16.626 \mathrm{MeV}$ state was made mainly to avoid conflict with the direct measurement of Ref. [14].

In summary, we have shown that the $16.8 \mathrm{MeV}$ state in ${ }^{9} \mathrm{~B}$ is unable to enhance the ${ }^{7} \mathrm{Be}(d, p)$ reaction rate by the amount needed to resolve the cosmological lithium problem. With the new precise determination of the energy of the $16.8 \mathrm{MeV}$ state [18], the reduction in ${ }^{7} \mathrm{Li}$ abundance owing to the inclusion of the resonant contribution of the $16.8 \mathrm{MeV}$ state to the ${ }^{7} \mathrm{Be}(d, p)$ reaction rate is at most $3.5(8) \%$ and probably much lower depending on the decay properties of the 16.8 MeV state which remain unknown. In line with Ref. 17], we conclude that all possibilities for solving the cosmological lithium problem by conventional nuclear physics means now seem to have been exhausted.

OSK acknowledges support from the Villum Kann Rasmussen Foundation. BD acknowledges support from the Natural Sciences and Engineering Research Council of Canada. TRIUMF receives federal funding via a contribution agreement through the National Research Council of Canada.
[1] R. V. Wagoner, W. A. Fowler, and F. Hoyle, Astrophys. J. 148, 3 (1967).

[2] E. Komatsu et al. (WMAP Collab.), Astrophys. J. Suppl. 192, 18 (2011).

[3] R. H. Cyburt et al., J. Cos. Astropart. Phys. 11, 012 (2008).

[4] For an example, see R. H. Cyburt et al., J. Cos. Astropart. Phys. 10, 021 (2009).

[5] A. J. Korn et al., Nature 442, 657 (2006).

[6] R. H. Cyburt, Phys. Rev. D 70, 023505 (2004).

[7] R. H. Cyburt and M. Pospelov, arXiv:0906.4373v1 [astro$\mathrm{ph}$ (2009).

[8] N. Chakraborty, B. D. Fields, and K. A. Olive, Phys.
Rev. D 83, 063006 (2011).

[9] D. R. Tilley et al., Nucl. Phys. A 745, 155 (2004).

[10] F. Ajzenberg-Selove, Nucl. Phys. A 506, 1 (1990).

[11] B. Pugh, Ph.D. thesis, Massachusetts Institute of Technology (1985).

[12] K. Kadija, G. Paić, B. Antolković, A. Djaloeis, and J. Bojowald, Phys. Rev. C 36, 1269 (1987).

[13] S. Dixit et al., Phys. Rev. C 43, 1758 (1991).

[14] C. Angulo et al., Astrophys. J. 630, L105 (2005).

[15] F. Hinterberger et al., Nucl. Phys. A 299, 397 (1978).

[16] P. von Brentano, Phys. Lett. B 246, 320 (1990).

[17] R. N. Boyd, C. R. Brune, G. M. Fuller, and C. J. Smith, Phys. Rev. D 82, 105005 (2010). 
[18] C. Scholl et al., Phys. Rev. C 84, 014308 (2011).

[19] L. H. Kawano, Report No. FERMILAB-Pub-92/04-A, preprint, 1992.

[20] M. S. Smith, L. H. Kawano, and R. A. Malaney, Astrophys. J. Suppl. Ser. 85, 219 (1993).

[21] D. D. Clayton, Principles of Stellar Evolution and $\mathrm{Nu}-$ cleosynthesis, New York, McGraw-Hill, 1968.

[22] A. M. Lane and R. G. Thomas, Rev. Mod. Phys. 30, 257 (1958).

[23] T. Teichmann and E. P. Wigner, Phys. Rev. 87, 123 (1952). 